\title{
Risk-Assessment for Equipment Operating on the Lunar Surface
}

\author{
R.C. Richmond ${ }^{1}$, A. Kusiak ${ }^{2}$, N. Ramachandran ${ }^{1}$ \\ ${ }^{I}$ NASA Marshall Space Flight Center, Huntsville AL 35812 \\ ${ }^{2}$ University of Iowa, Department of Mechanical and Industrial Engineering, Iowa City IA 52242
}

\begin{abstract}
Particle-size distribution of lunar dust simulant is evaluated using scanning electron spectroscopy in order to consider approaches to evaluating risk to individual mechanical components operating on the lunar surface. Assessing component risk and risk-mitigation during actual operations will require noninvasive continuous data gathering on numerous parameters. Those data sets would best be evaluated using data-mining algorithms to assess risk, and recovery from risk, of individual mechanical components in real-time.
\end{abstract}

Keywords: lunar dust; SEM image analysis; mechanical testing; wear; risk-assessment

PACS: 81.70.-q: Methods of materials testing and analysis

\section{INTRODUCTION}

NASA commitment to return to the moon for long-term habitation and exploration provides new needs for riskassessment of mechanical components associated with habitation and exploration. Constraints for assessing risk during lunar exploration are that mechanical problems will often need be detected, evaluated, and mitigated before associated components fail, given that component change-out will be limited as a method to resolve risk. Thus, providing prospective risk-assessment on individual mechanical components is needed to achieve effective missioncritical risk-resolution necessary for lunar exploration.

Deterministic risk-assessment was largely used as a retrospective evaluation of potential failure until the Challenger accident in 1986, after which NASA mandated use of retrospective probabilistic risk-assessment of engineering systems to better incorporate variables and uncertainties of operational parameters that could affect outcomes of failure (Bedford and Cooke, 2000). Now, risk-assessment of individual mechanical components used in lunar exploration will likely need to rely on prospective real-time evaluations of critical components during actual operation in order to detect departure from normal operations, and thereby allow repair prior to component failure.

Particle-size-dependent distributions of lunar dust on operating surfaces and within lubricants of operating mechanical components, and subsequent consequences of wear and fouling on operations of those components, are uncertain. Arguably, the highest priority for initiating risk-assessments posed by lunar dust to mechanical and passive components is to observe and analyze dust particle distributions upon and within those components during actual operations on the lunar surface, in association with projected outcomes such as increasing thermal load, torque, etc.

Scanning electron microscopy (SEM) can be applied for evaluating distributions of lunar simulant JSC-1AF (Oribital Technologies Corporation, 2006) on component surfaces, where those distributions on and within mechanical components and materials can provide baseline data used to detect departure from normality of operations in the lunar environment. SEM can also sense dust particles within ca. 100 micron of greases used on components such as bearings and gears, thereby informing on the presence and fate of lunar dust simulant within that operational environment. We will demonstrate how visualization of lunar dust simulant by SEM may contribute to a baseline database for referencing prospective risk-assessment of individual components, a reasonable approach to anticipating, and reacting to, mission-critical and life-critical problems prior to terminal crisis. 


\section{METHODS AND RESULTS}

The ability of SEM to characterize particle size distributions of lunar dust and the dust simulant JSC-1AF was recently presented (Taylor, 2007). This characterization was important in part because particle-size distributions were determined using scanning electron microscopy (SEM) for two Apollo return samples of actual lunar dust. As anticipated from earlier numerous descriptions of lunar dust, the average particle size for lunar dust was indeed quite small, specifically about 0.1 micron and 0.3 microns for return samples \#10084 and \#70051, respectively, whereas the average size for the currently available fine lunar dust simulant JSC-1AF was determined to be ca. 0.7 micron. Those dust samples were prepared for SEM analysis with rigorous separation of individual particles (Park et al., 2006).

Using an FEI Quanta 600 FEG field-emission SEM, we have also imaged JSC-1AF lunar dust simulant with preliminary intent to observe its presence on, and interaction with, mechanical components in order to provide that data then to assessments of risk to those components. We have verified the fine particle-size distribution reported by Dr. Taylor for JSC-1AF, but using sample preparation simply prepared by spreading a $1 \mathrm{mg}$ sample of JSC-1AF simulant upon a weak ceramic magnet, which facilitated spreading, followed by direct transfer of the entire sample to standard carbon tape that was attached then to an SEM stub. This simple sample preparation was chosen because such a direct retrieval method will be required when obtaining in situ samples of dust from component surfaces of components for subsequent SEM analyses. Results are shown in Figure 1, where particle density is great enough to interfere somewhat with determination of particle-size distribution obtained by image analysis, leading to a mean particle size about two-fold greater than the 0.7 micron previously reported (Taylor, 2007).

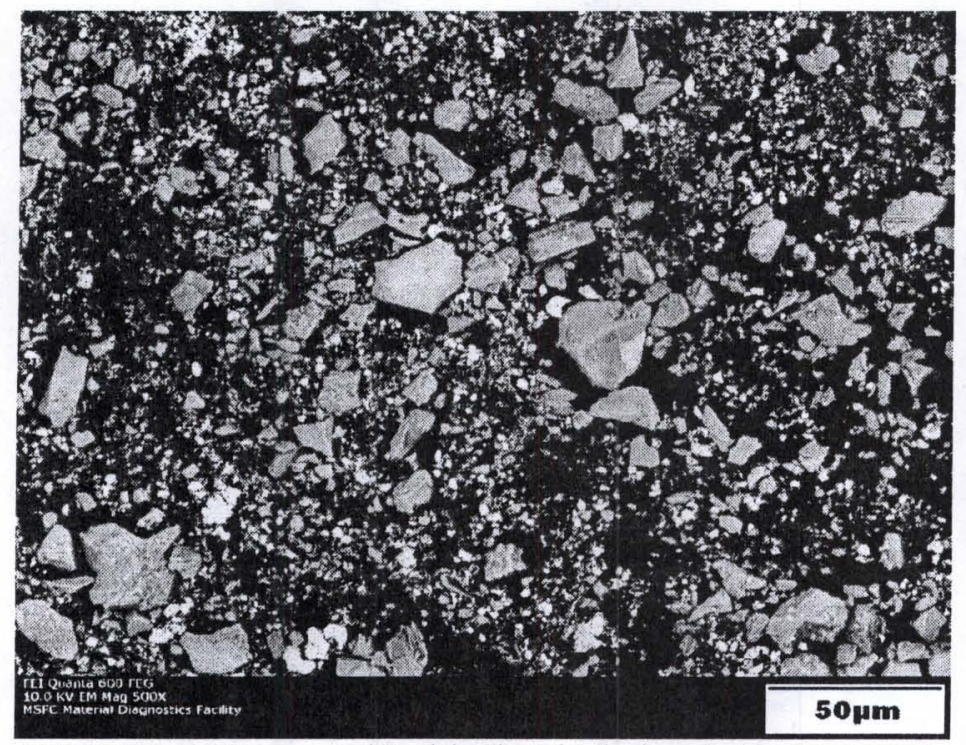

\begin{tabular}{|l|r|}
\hline Particle count & 2535 \\
\hline Dmin, $\mu \mathrm{m}$ & 0.72 \\
\hline Dmax, $\mu \mathrm{m}$ & 32.52 \\
\hline Davr, $\mu \mathrm{m}$ & 1.67 \\
\hline D std. dev., $\mu \mathrm{m}$ & 1.75 \\
\hline
\end{tabular}

FIGURE 1. SEM Image and Particle-Size Distribution. Carbon Tape Transfer of JSC-1AF from Ceramic-Magnet 500X.

The magnified image in Figure 2 of JSC-1AF from the same sample used for Figure 1 shows the presence of very small (ca. $50 \mathrm{~nm}$ diameter) particles in the JSC-1AF dust simulant, and that two subpopulations of electrondiffraction images exist. The grey subpopulation indicates lower energy electron emission, and the bright subpopulation indicates higher energy electron emission, where in SEM this typically indicates lighter versus heavier elemental content, respectively. Indeed, energy dispersive spectroscopy (EDS) of bright particles reveals composition suggesting magnetite as a predominant mineral, and for grey particles plagioclase feldspar as a predominant mineral, as shown in Figures 3 and 4, respectively. This is an alert for methods of risk-assessment . that may be developed for mechanical components based upon mineralogic compositions found in componentassociated dust -- for example, one mineral type may prove to be more abrasive or material-interactive than another mineral type, and specifically here Mohs' hardness for plagioclase is 6.0, but 5.0 for magnetite (READE, 2007). 


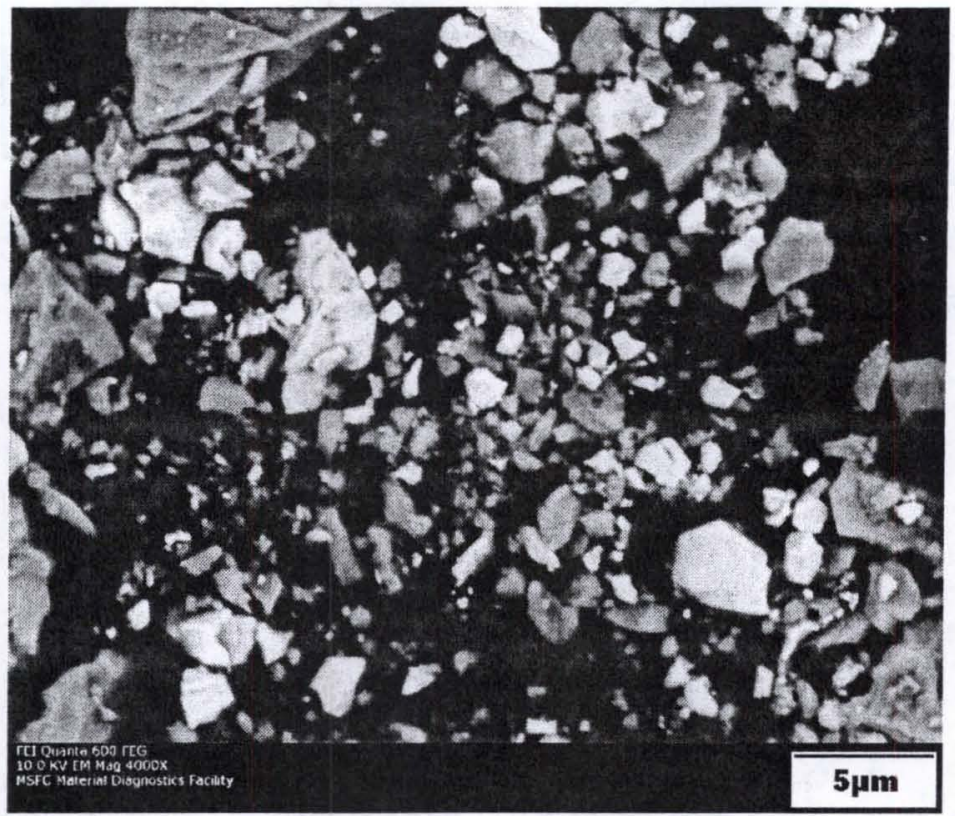

FIGURE 2. SEM Image. Carbon Tape Transfer of JSC-1AF from Ceramic-Magnet 4000X.

Anticipating the need to analyze dust from component surfaces not accessible directly to standard SEM dust sample preparation, e.g., the internal race of a bearing, a JSC-1AF sample was recovered from the same weak ceramic magnet surface using the same sample preparation providing Figures 1 and 2, only this time using the lens cleaner First Contact (formerly called Opticlean), rather than carbon tape, that is touted for total dust particle removal from lens surfaces (Bennett, 2000). First Contact is a low-surface tension solvent that dries to a transparent film investing dust particles that are then stripped from the surface when the film is peeled off. The peeled film, regardless of film thickness, was thought likely to provide an undersurface that exposes the actual dust particles to analyses by SEM. This assumption was tested and found correct, i.e., adequate imaging of JSC-1AF particles on the undersurface of First Contact film recovered from the same ceramic magnet surface used for Figures 1 and 2 provided the SEM images of Figures 5 and 6.

The grinding of dust within mechanical components such as bearings and gears likely will reduce its particle size, and that reduction may be usefully evaluated for indirectly estimating wear of that component. Therefore, JSC-1AF simulant was briefly ball-milled in a zirconia ceramic mill, providing then in Figure 6 the image of a much reduced particle size, but with substantially increased clumping of particles compared to the direct preparation of JSC-1AF in Figure 1, where such aggregation for the purpose of simulant testing may be somewhat analogous to the aggregation of dust particles often reported for lunar dust (e.g., Taylor, 2007; Walton, 2007). The latter difference is useful regards the concept that components such as bearings and gears at times may grind up the generally semi-hard (ca. Mohs' scale 5-7) lunar dust simulant with associated component wear problems.

The use of SEM in Figure 7 demonstrates elemental mapping to analyze the presence of JSC-1AF dust particles ( $5 \%$ by weight in this case) within low vapor pressure Braycote grease currently used in space applications, in order, for example, to compare before versus during component operations. The approach was to use incident high-energy electrons (30 kV in this case), and then map the return of characteristic Xrays, both directions thereby providing relatively good energy penetrations for seeing dust particles within grease to the depth of several microns. Direct SEM of this JSC-1 AF grease mix is shown initially (panel 1) and after $30 \mathrm{~min}$ of electron beam application (panel 2). Elemental mapping of the sample field of panel 1 is shown for $\mathrm{C}, \mathrm{O}, \mathrm{F}, \mathrm{Al}, \mathrm{Si}, \mathrm{S}, \mathrm{Ca}$, and $\mathrm{Fe}$ in panels 4 through 11, respectively. The composite of this elemental mapping is shown in panel 3. 


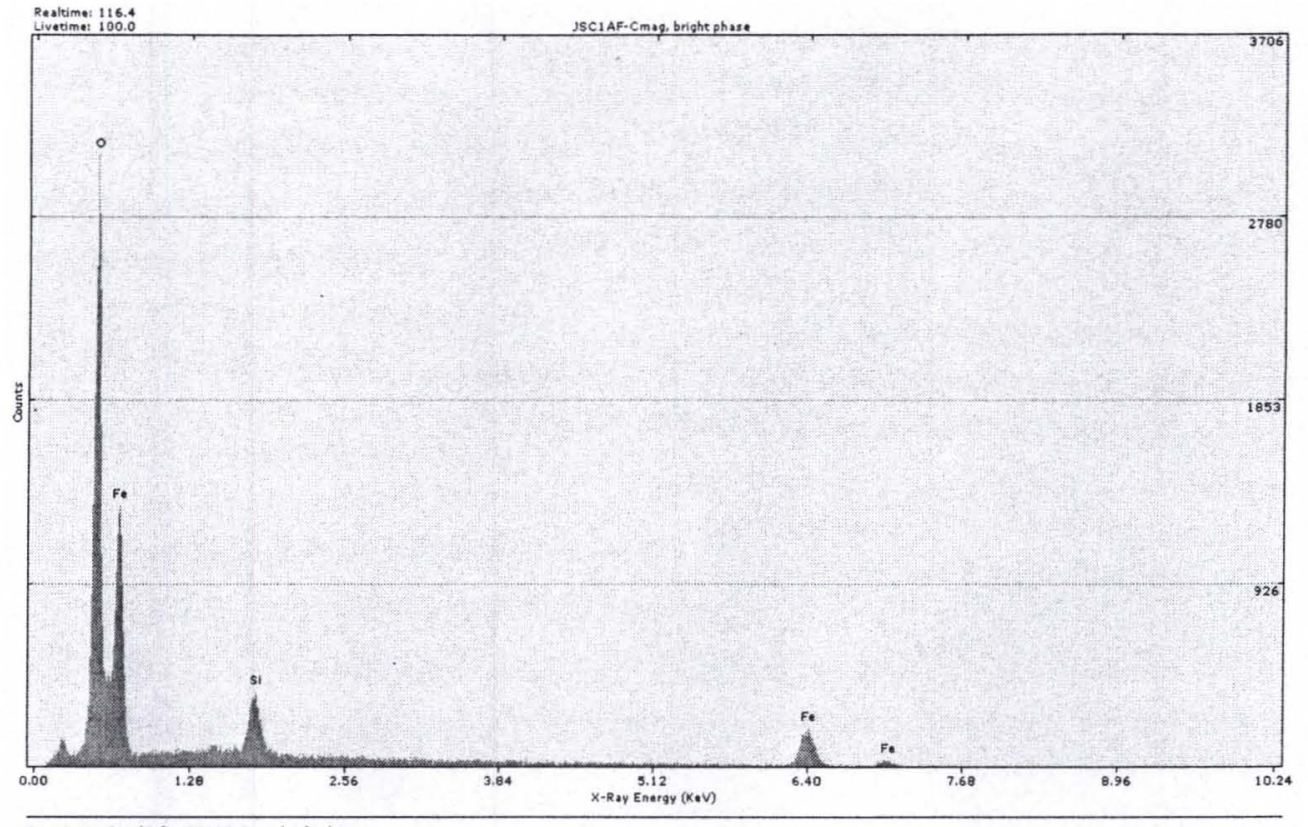

Quancitative Results for ISC1 AF-Cmag, bright phase Analysis: Bulk Method, Standardless
Acquired 25-Jul-2007, $10.0 \mathrm{KeV} @ 10$.V/channel

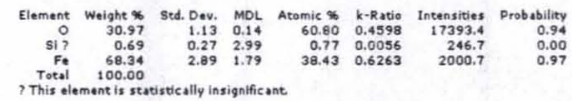

FIGURE 3. EDS Profile. Bright Phase; Carbon Tape Transfer Sample; JSC-1AF 4000X.

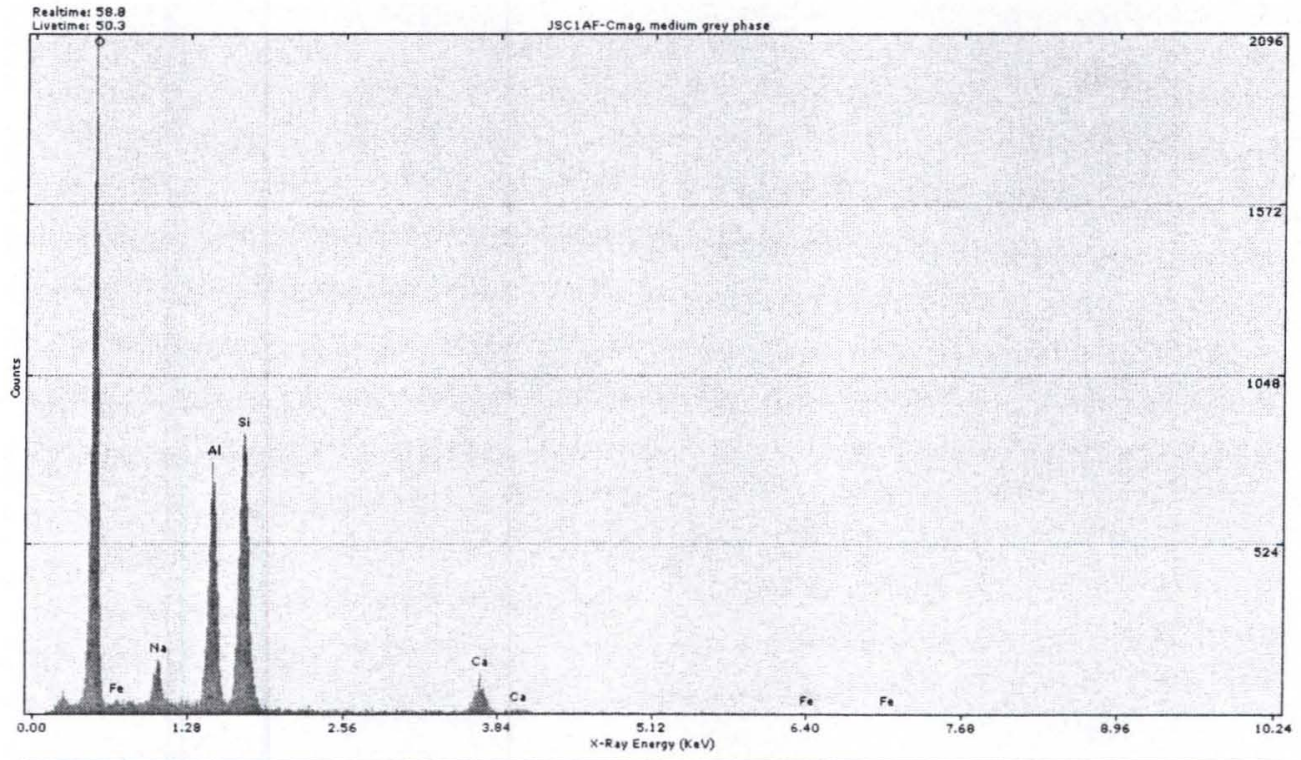

Quantireive Results for JSC1AF-Cmaq medium grey phase

Analysist Bulk Mechod: Standardless
Aceuired 25-Jul-2007, $10.0 \mathrm{~K}$ ov 10 . Vichannel

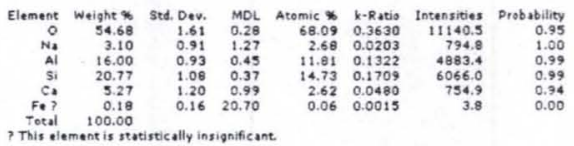

FIGURE 4. EDS Profile. Grey Phase; Carbon Tape Transfer Sample; JSC-1AF 4000X. 


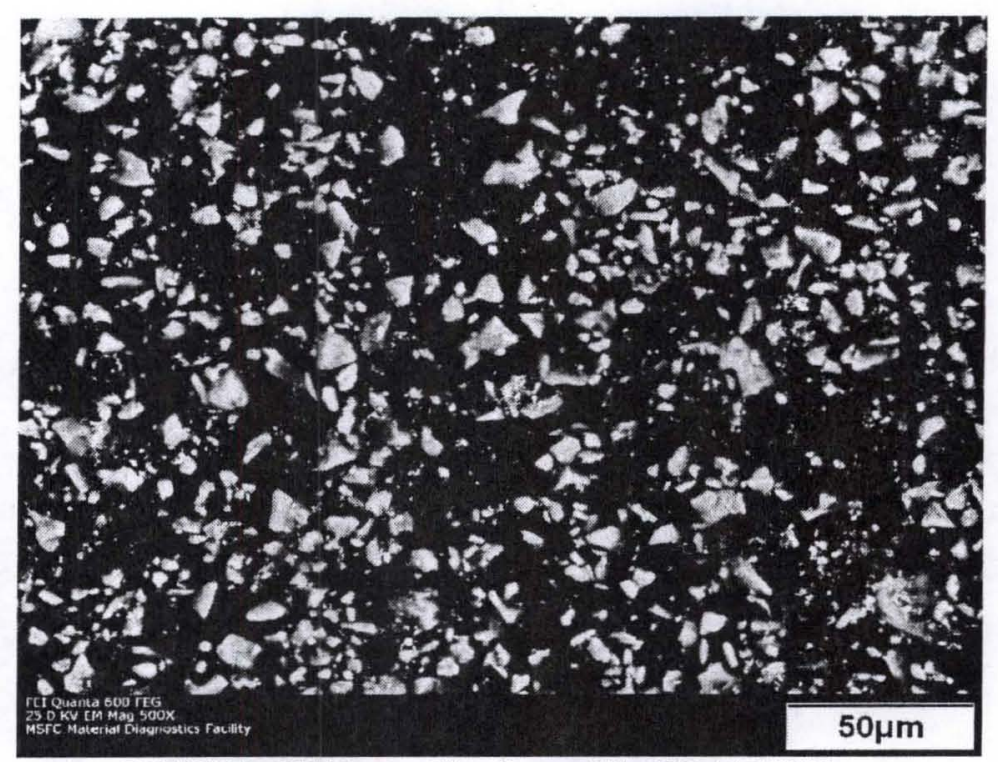

FIGURE 5. SEM Image. First Contact Film JSC-1AF 500X.

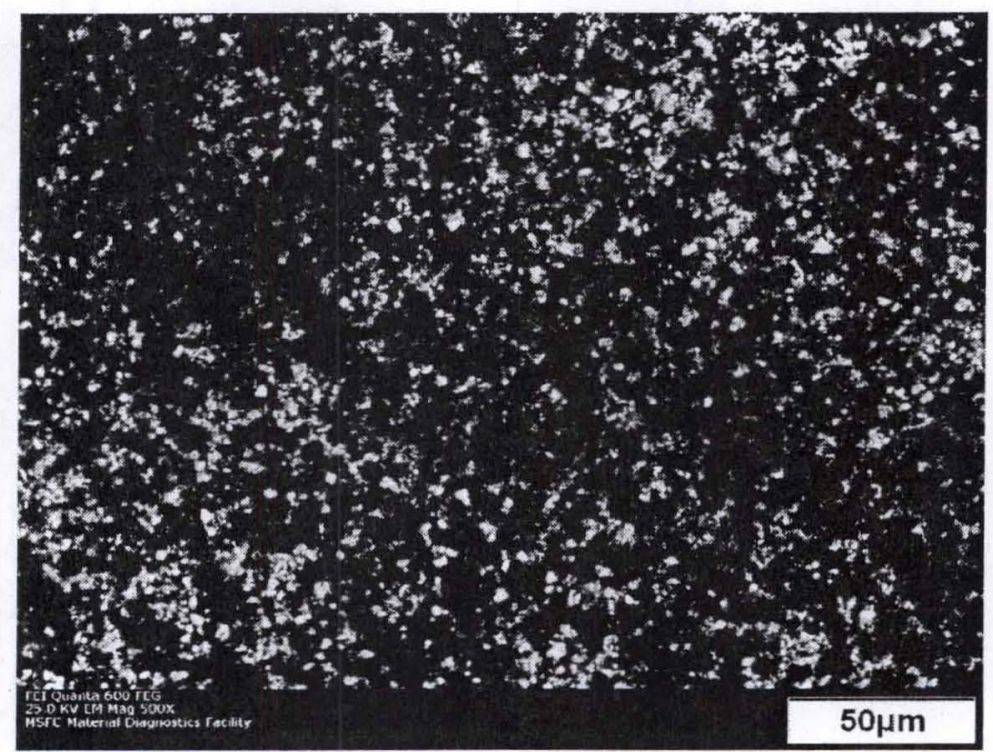

FIGURE 6. SEM Image. First Contact Film JSC-1AF Ball-Milled 500X.

A few useful comments in support of this method for testing dust particles in grease are the following:

1. extended electron beam exposure on this region-of-interest (ROI) leads to ablation/evaporation of grease, which provides improvement of direct image analysis of invested dust particles in grease, as shown in panel 1 (initial SEM image) compared to panel 2 (30 min of beam time prior to SEM image) of Fig 7;

2. the low vapor pressure perfluorocarbon-based Braycote grease used by NASA in high-vacuum environments is shown in panel 6 (fluorine) to be of smooth composition;

3. the Si map image of panel 8 is best used for determining particle size distributions in non-Si containing greases;

4. the type of mineral in a particle is inferred by its elemental map, and the degree to which that particle survives operational wear can be tracked in the post-operation comparison; as needed, the mineralogy-related EDS composition can be recovered at any time since that information is available within each region-of-interest (ROI) of any elemental map image. 

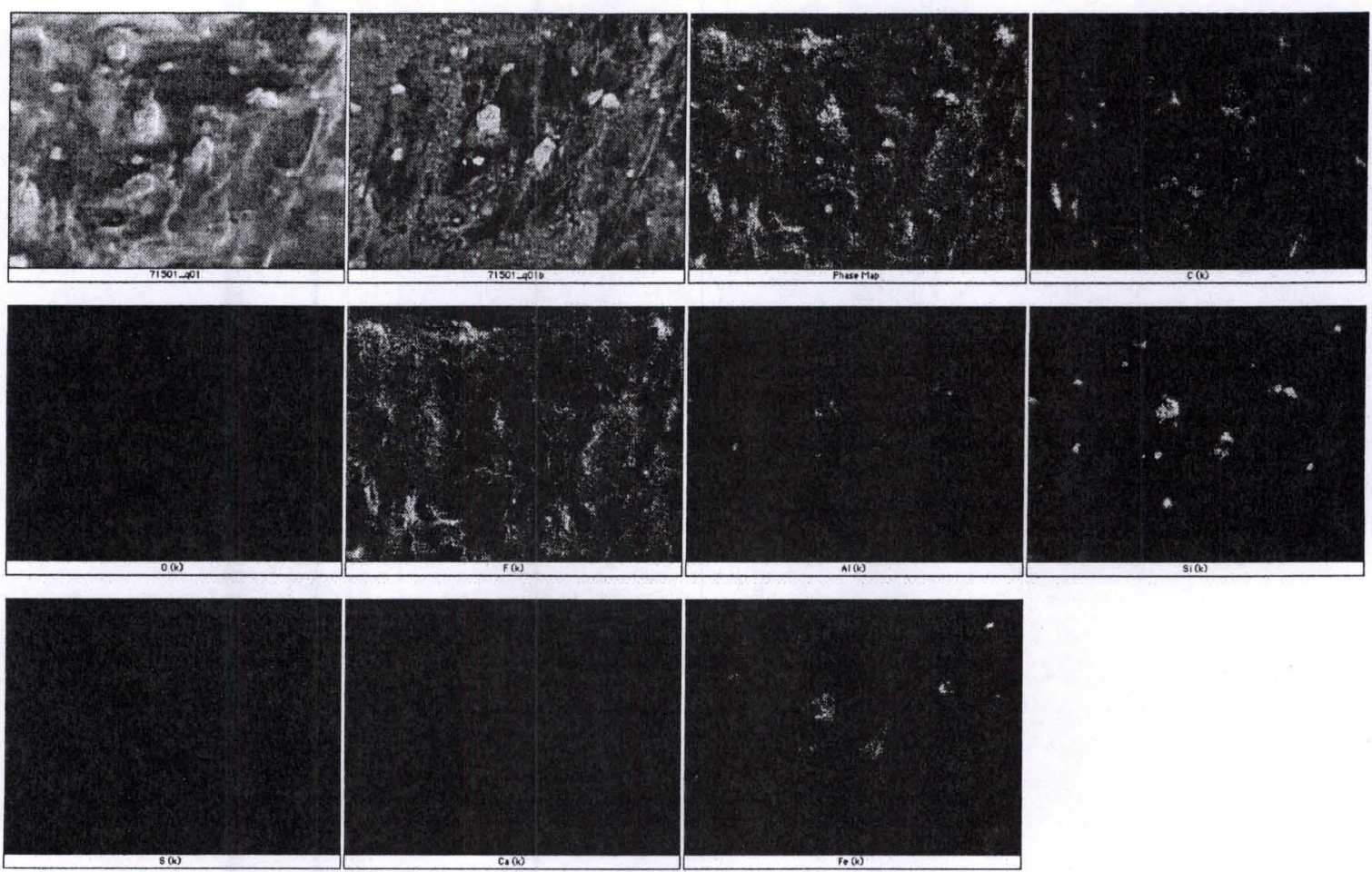

FIGURE 7. SEM Direct and Elemental Mapping of Braycote Grease and JSC-1AF.

It is important to note that use of element-map imaging demonstrated in Figure 7 for JSC-1AF particles in grease is complementary to the previously demonstrated use of First Contact polymer-film retrieval of JSC-1AF particles from material surfaces for direct SEM image analysis.

In conclusion, SEM is adequately established for evaluation of dust particles on component surfaces directly, and in high-vacuum greases used in individual components of lunar exploration, before and during component operations in order to achieve useful analyses of wear processes caused by lunar dusts and simulants. Whereas SEM is an adequate Earth-based method for some dust visualizations and analyses on component surfaces, it will not be adequate for use during actual lunar exploration. Rather, a photonic dust-particle inspection microscope must be developed for similar visualizations and analyses of components during their operations on the lunar surface; nonetheless, the applications of this type of inspection data to individual prospective risk-assessments that we propose to be necessary will not change.

\section{DATA REACTIVE PARADIGM}

Paradigms of both deterministic and probabilistic risk-assessments are strictly retrospective in design. As such, neither provides analysis of risk for individual components during actual operation; they only provide uncertainties, typically huge, of failure forecast for individual components. It is required to preserve function of individual components operating on the lunar surface for no other needed reason than the fact those components cannot be routinely changed out in the event of failure. It is therefore also required to apply a paradigm of prospective riskassessment to these individual components in real-time during operations. Considerations necessary for this paradigm are introduced here.

\section{Risk Planning Stage}

1. Obtain initial observations, including dust particle-size distributions on surfaces and in greases, of mechanical components in order to establish a baseline database prior to start of operation in the lunar environment. 
2. Reference operational observations to the baseline database, which would include dust particle distributions plus other parameters, such as particle-wear profiles matched to material and component wear patterns retrospectively established from earlier tribologic testing.

3. Iteratively collect data sets from components over time of operation in order to validate compliance with, or departure from, the initial baseline database.

\section{Risk Identity Stage}

1. Repeated comparisons with the baseline database will collapse uncertainties around individual mechanical components that depart from baseline during actual operations.

2. Collect additional data from an affected component in order to provide risk-assessment for that individual component.

\section{Risk-management Stage}

1. Detailed analysis of an individual mechanical component identified for high prospective risk of problem/failure will provide information from which specific mitigation can be identified in order to return that component to riskfree continuing use.

2. The suite of data-gathering and data-analysis tools used to provide prospective iterative data sets for riskassessment will also be used to manage the real-time mitigation, i.e., return to baseline, of individual mechanical components.

\section{Data Mining for Assessing Risks as Departures from Baseline and Returns to Baseline}

Data-mining algorithms can be used to analyze complex mixtures of component wear and failure modes. Noisy and temporal data anticipated at times with prospective individual component risk-assessment can be handled by the use of bidirectional mining. Data-mining algorithms can be used to analyze component wear and failure modes.

\section{Data Analysis with Data-Mining Algorithms}

Data mining encompasses a set of algorithms for revealing hidden patterns and extracting useful knowledge from data. Classes of these algorithms that are of potential value to individual prospective risk-assessment have been listed (Kusiak, 2001; Kusiak et al., 2000), with some being:

a. Classical statistical methods (e.g., linear and quadratic discriminant, and logistic discriminant, analyses);

b. Modern statistical techniques (e.g., projection pursuit classification, density estimation, $k$-nearest neighbor, casual networks, Bayes algorithm);

c. Neural networks (e.g., backpropagation, linear vector quantifiers, and radial basis function networks);

d. Decision tree algorithms

e. Association rule algorithms

f. Learning classifier systems.

In essence, these algorithms are integrated to learn from the data (through iterative training data sets); however, the form of the extracted knowledge (patterns) varies. Some algorithms provide explicit knowledge (e.g., decision tree algorithms) or implicit models (e.g., Bayes algorithm). In addition, learning, as autonomous pattern discovery, can be supervised (e.g., backpropagation neural network) or unsupervised (e.g., $k$-nearest neighbor algorithm). Supervised learning labels the data, while unsupervised learning deals with unlabeled data. Both supervised and unsupervised algorithms would be combined in continually revising uncertainties of increasing risk, as well as decreasing risk after mitigation, in an operating individual component.

\section{Bidirectional Data Mining}

Most data-mining algorithms establish associations among individual features (horizontal mining). However, for individual risk-assessment in operational real-time, there is likely value to consider both horizontal and vertical feature functions (Kusiak, 2001). The concept of bidirectional mining appears to be a viable method for generating derived features. 
Examples of horizontal functions to be considered include:

a. Logic expression of parameters Fi, Fj, ... Fn, where the <logic operator $>=\{$ AND, OR, NOT, EXOR $\}$

b. Arithmetic expression of parameters $\mathrm{Fi}, \mathrm{Fj}, \ldots \mathrm{Fn}$, where the <arithmetic operator $>=\left\{+,-, /, \times, \sqrt{ },{ }^{\mathrm{n}}, \mid\right\}$

c. Phenomena-based expressions related to performance prediction.

Examples of vertical functions to be considered include:

a. Wavelets of single and multiple parameters.

b. Data and user derived events.

c. Fourier transforms.

d. Kernel functions.

The need for an algorithm that generates feature functions is twofold. First, feature functions may serve as partial models, including science-based models. These partial models may be integrated with the previously unknown relationships discovered by data-mining algorithms in a usable model. The feature functions can provide a mechanism for comprehending dynamic risk. Second, feature functions have been used in other computational intelligence tools, e.g., neural networks, where each neuron represents a quantitative function.

Recent progress in data analysis has led to notable applications of data-mining algorithms in product development (Kusiak and Salustri, 2007), mass customization (da Cunha, Agard and Kusiak, 2007), discovery of emerging faults (Kusiak and Shah, 2006), and genetics (Shah and Kusiak, 2007). These applications exhibit characteristics similar to dynamic individual risk discussed here for operational mechanical components.

Algorithms used by data mining for risk-assessment of individual components require gathering data in real-time from parameters meaningful for database comparisons and iterative training-set progressions. Table 1 provides examples of multiple non-invasive data gathering in real-time required from a few of the multiple parameters anticipated to inform on risk-development, as well as risk-recovery, for individual mechanical components during operation on the lunar surface.

TABLE 1. Parameter Data for Real-Time Individual Component Operational Data Acquisition.

\begin{tabular}{|c|c|c|c|c|c|c|c|}
\hline $\begin{array}{l}{ }^{*} \text { Time } \\
\text { Stamp }\end{array}$ & $\begin{array}{l}\text { Dust } \\
\text { Presence }\end{array}$ & $\begin{array}{l}\text { Dust } \\
\text { Particle }\end{array}$ & Torque & $\begin{array}{l}\text { Thermal } \\
\text { Load }\end{array}$ & $\begin{array}{l}\text { Vibration } \\
\text { Level }\end{array}$ & $\begin{array}{l}\text { Power } \\
\text { Level }\end{array}$ & $\begin{array}{l}\text { Tribo- } \\
\text { Charging }\end{array}$ \\
\hline $\begin{array}{l}\text { 1. Lunar } t-1 \\
\text { 2. Lunar } t-2 \\
\text { 3. Lunar } t-n\end{array}$ & ok & ok & ok & ok & ok & ok & ok \\
\hline $\begin{array}{l}\text { A. Lunar } \mathrm{t}-\mathrm{n} 1 \\
\text { B. Lunar } \mathrm{t}-\mathrm{n} 2 \\
\text { C. Lunar } \mathrm{t}-\mathrm{nx}\end{array}$ & ${ }^{* *} \mathrm{ob} ?$ & ob? & $\mathrm{ob} ?$ & ob? & $\mathrm{ob} ?$ & $o b ?$ & ob? \\
\hline $\begin{array}{l}\text { a. Lunar t-nx1 } \\
\text { b. Lunar } \mathrm{t}-\mathrm{n} \times 2 \\
\text { c. Lunar t-nxy }\end{array}$ & $* * *$ bib & bib & bib & bib & bib & bib & bib \\
\hline
\end{tabular}

*1-3: Parameters within baseline

A-C: Parameter(s) outside baseline and risk determined

a-c: Mitigation applied and parameters returned to baseline

**ob: out-of-baseline

***bib; back-in-baseline 


\section{CONCLUSION}

An approach using imaging for monitoring lunar dust particle distributions in individual mechanical components operating on the lunar surface has been suggested here. More useful, however, is that considering this currently meager knowledge of dust particle distribution relative to assessing risks amplifies the need for obtaining extended data sets in order to achieve adequate real-time risk-assessment of mechanical components during lunar operations.

Raising the need for real-time data gathering from individual components also raises the issue of how to evaluate that data to achieve prospective risk-assessment of individual components prior to catastrophic failure. The most likely success of that objective resides in analyses of data using flexible algorithms of data mining, including selfteaching algorithms. Computationally, this is largely a machine-autonomous approach useful for minimal crew involvement, i.e., a priority for manned lunar exploration.

The process of failure for mechanical components on the lunar surface will be nonlinear and nonstationary. A testing procedure is needed to first, collapse by real-time data acquisition the huge retrospective uncertainty of risk associated with each individual component, and second, validate and/or guide optimization of recovery of an individual component as intended for any specific mitigation procedure. The variable, unpredictable, and complex data anticipated for use in this testing process will require test procedures that are inherently flexible and selfteaching so that autonomous decisions can be made in selection and applications of available algorithms for riskassessment and risk-management (Kusiak and Song, 2006). This is the province of data mining.

In comparison to basic science models, data mining offers advantages in optimizing risk-assessment by considering many parameters and generating accurate results. As examples, some data-mining algorithms produce explicit knowledge that is obviously understandable to a user, and some do not; some utilize iterative data sets for training purposes, thus refining choice and accuracy of algorithms used for assessment, such as neural nets; some provide decision-trees wherein independent parameter values are used to determine decisions, i.e., values of the dependent variables. The numerous algorithms available to data mining all require baseline, i.e., historical, data sets plus prospective operational data sets in order to provide individual risk-assessment and risk-management in real-time. The kinds of data required from mechanical components in order to assess lunar dust-related risks will include the kind of introductory knowledge of dust particle distributions introduced here, plus data from several other tribologic and operational parameters. Some of these related parameters are obvious, such as torques, thermal loads, power efficiencies, material wear indicators, etc.; some parameters may only be identified during operations as a consequence of data-processing from data-mining algorithms, given currently unknown consequences of lunar thermal cycling, long-term effects of radiation quality and exposures, highly variable electrostatics, etc., all combined with an unremitting high-vacuum lunar environment.

In reality, several additional parameters related to Table 1, both anticipated and unanticipated, will need real-time operational data acquisition for each mechanical component designated as life-critical and/or mission-critical in order to collapse risk uncertainties in time for rescue by mitigation procedures. Some of these parameters will be controllable or adjustable, such as those affected by dust exclusion, torque, triboelectric charging (Sternovsky, Robertson, Sickafoose, 2002), dust plume generation in the working lunar environment, etc., and some will not be controllable, such as photoelectric charging (Stubbs, Vondrak and Farrell, 2005), thermal cycling, solar wind, highvacuum effects, etc. Changeable and temporal patterns of data amongst these multiple parameters can be adequately managed by the available dynamic tools and algorithms of data mining mentioned above, and this management can be performed largely with machine-based autonomy.

In closing, it is stressed that a number of noninvasive reporting sensors and systems will be needed to provide photonic images and physical data sets from critical components during real-time operation, not only to alert individual failure, but also to track mitigation for return to normal operation. The commercial inventory required to develop these sensors is nearly complete now. It would be incumbent upon reasonable planning for lunar exploration to begin advancing the fabrication of these sensors and systems at this time. 


\section{ACKNOWLEDGMENTS}

The Lunar Dust Assessment Project of the Mechanical Systems SPRT funded through the NASA. Engineering and

Safety Center is acknowledged for partial support of this work.

\section{REFERENCES}

Bedford, T., and Cooke, R., Probabilistic Risk Analysis: Foundations and Methods, Cambridge University Press, Cambridge, 2000, pp. 4-5.

Bennett, J.M., and Rönnow, D., "Test of Opticlean Strip Coating Material For Removing Surface Contamination", Appl Optics, $39: 2737-2739(2000)$.

da Cunha, C., Agard,B., and Kusiak, A., "Design for Cost: Module-Based Mass Customization," IEEE Trans in Automation Sci Engineer, 4:350-359 (2007).

Kusiak, A, Feature transformation methods in data mining, IEEE Trans Electronics Packaging Manufacturing, 24:214-221 (2001).

Kusiak, A., Kern, J.A., Kernstine, K.H., and Tseng, T.L., Autonomous decision-making: a data mining approach, IEEE Trans Information Technol Biomed, 4:274-284 (2000).

Kusiak, A., and Salustri, F.A., "Computational Intelligence in Product Design Engineering: Review and Trends, "IEEE Trans on Systems, Man, and Cybernetics: Part C, 37(5): 766-778 (2007).

Kusiak, A., and Shah, S., "A Data-Mining-Based System for Prediction of Water Chemistry Faults," IEEE Trans Indust Electronics, 53(2): 593-603 (2006).

Kusiak, A., and Song, Z., "Combustion Efficiency Optimization and Virtual Testing: A Data mining Approach,", IEEE Transact Indust Informatics, 2:176-184 (2006).

Orbital Technologies Corporation (ORBITEC), "Characterization summary of JSC-1 AF lunar mare regolith simulant," (2006), www.lunarmarssimulant.com/catalog/docs/JSC-1AF\%20Characterization.pdf, November 15, 2006.

Park, J., Liu, Y., Kihm, K.D., Hill, E., and Taylor, L.A, "Submicron Particle Size Distribution of Apollo 11 Lunar Dust," Proceedings Earth \& Space 2006: Engineering, Construction, and Operations in Challenging Environment, Houston TX, March 5-8, 2006.

READE , "Mohs' Hardness of Abrasives", Review of Abrasives (2005), http://www.reade.com/Particle_Briefings/mohs_hardness_abrasive_grit.html, February 8, 2007.

Shah, S., and Kusiak, A., "Cancer Gene Search with Data-Mining and Genetic Algorithms," Computers Biol Med, 37(2) 251-261 (2007).

Sternovsky, Z,, Robertson, S., Sickafoose, A., Colwell J., and Horányi, M., "Charging of lunar and Martian Dust Simulants", $J$. Geophys. Res., 107(E11):15.1-15.8 (2002).

Stubbs, T. J., Vondrak, R. R., and Farrell, W. M., "A Dynamic Fountain Model for Lunar Dust," Lunar and Planetary Science, XXXVI:1-2 (2005).

Taylor, L., "Everything you ever wanted to know about lunar dust," NASA Advisory Council Workshop on Science Assaciated with the Lunar Exploration Architecture, Tempe AZ; February 27-March 2 (2007), http://www.lpi usra.edu/meetings/LEA/presentations/, June 3, 2007.

Walton, O.R., "Adhesion of Lunar Dust," NASA Report: NASA/CR-2007-214685, National Technical Information Service, Springfield VA, 2007. 